\title{
Photonuclear reactions of light nuclei studied with high-intensity real photon beams
}

\author{
Tatsushi Shima* \\ Research Center for Nuclear Physics, Osaka University \\ E-mail: shimaercnp.osaka-u.ac.jp
}

\begin{abstract}
Photonuclear reactions of light nuclei play crucial roles in various processes of nucleosynthesis in stars and in the early universe. They are also important in relation to the explosion mechanism of Type-II supernovae and associating neutrino-induced nucleosynthesis, because they can provide useful information about the analogous processes of the neutrino-induced nuclear reactions. A new experimental method by means of a laser-Compton scattered $\gamma$-ray beam together with an active-target technique has been developed for studies of photonuclear reactions relevant for nuclear astrophysics. The method has been successfully used for high-precision measurements of the absolute cross sections of the photodisintegrations of ${ }^{4} \mathrm{He}$ and ${ }^{12} \mathrm{C}$ in the $\gamma$-ray energy range up to $30 \mathrm{MeV}$, where serious discrepancy has existed among the previous data. In this contribution the new experimental results on the photonuclear reactions of ${ }^{4} \mathrm{He}$ and ${ }^{12} \mathrm{C}$ and a new $\gamma$-ray source at the NewSUBARU synchrotoron radiation facility will be presented.
\end{abstract}

International Symposium on Nuclear Astrophysics - Nuclei in the Cosmos - IX

25-30 June 2006

CERN

* Speaker. 


\section{Introduction}

Nuclear electromagnetic processes of radiative-capture reactions and photodisintegrations of light nuclei are considered to play crucial roles in various processes of nucleosynthesis occurring in stars and in the early universe. For quantitative studies of those nucleosynthesis processes, reliable data of the relevant nuclear reactions are indispensable. Photonuclear reactions will provide useful tools to obtain information about nuclear electromagnetic processes of many-body systems as well as of two-body ones. Also they would be useful to study the analogous processes of the neutrino-induced nuclear reactions [1], 2], which are considered to play critical roles in the dynamics of Type-II supernova explosions (SNes) [3, 4, 5] and in neutrino-induced nucleosynthesis [6, 7].

Recent developments of quasi-monochromatic $\gamma$-ray sources and highly sensitive particle detectors provide unique opportunities to perform high-precision measurements on nuclear reactions caused by low-energy $\gamma$-rays. In this paper recent experimental work on photonuclear reactions of ${ }^{4} \mathrm{He}$ and ${ }^{12} \mathrm{C}$ and a new high-performance $\gamma$-ray source at the NewSUBARU synchrotron radiation facility will be shown.

\section{Experimental method}

The cross section $\sigma$ of a photonuclear reaction is experimentally obtained as

$$
\sigma=\frac{R}{\varepsilon \cdot n \cdot L \cdot \Phi_{\gamma}}
$$

using measured values of the counting rate $R$, number density $n$ of the target nucleus, effective length $L$ of the target, efficiency $\varepsilon$ of the detector, and incident $\gamma$-ray intensity $\Phi_{\gamma}$. To measure the cross section accurately, one has to reduce the systematic errors of $n, L \varepsilon$ and $\Phi_{\gamma}$ as well as the statistical error of $R$. Therefore we designed a new experimental method as shown in Fig. 1.

\section{Laser Compton-scattered $\gamma$-ray beam}

A laser Compton-scattered (LCS) $\gamma$-ray beam is generated by head-on collision of a laser light and an electron beam circulating in a storage ring. The $\gamma$-ray energy $E_{\gamma}$ is given by the kinematical condition of the Compton scattering as

$$
E_{\gamma}=\frac{4 E_{\text {laser }} \cdot \gamma^{2}}{1+\gamma^{2} \theta^{2}+4 E_{\text {laser }} \cdot \gamma / m_{e} c^{2}},
$$

where $E_{\text {laser }}, \gamma$, and $\theta$ are the energy of laser photons, the relativistic factor of the electron, and the scattering angle of the photon, respectively. $m_{e}$ denotes the rest mass of the electron. By selecting the $180^{\circ}$ backward component of the scattered photons using a $\gamma$-ray collimator, a quasi-monochromatic $\gamma$-ray beam is obtained. Polarized $\gamma$-rays can be easily generated by using a polarized laser light. A LCS $\gamma$-ray beam has the following features;

- quasi-monochromatic $\left(\Delta E_{\gamma} / E_{\gamma}=5-10 \%\right)$ and variable (a few $\sim$ a few tenth $\mathrm{MeV}$ ) energy,

- little background $\gamma$-rays due to bremsstrahlung,

- small angular dispersion $(\Delta \theta<\sim 0.3 \mathrm{mrad})$, 


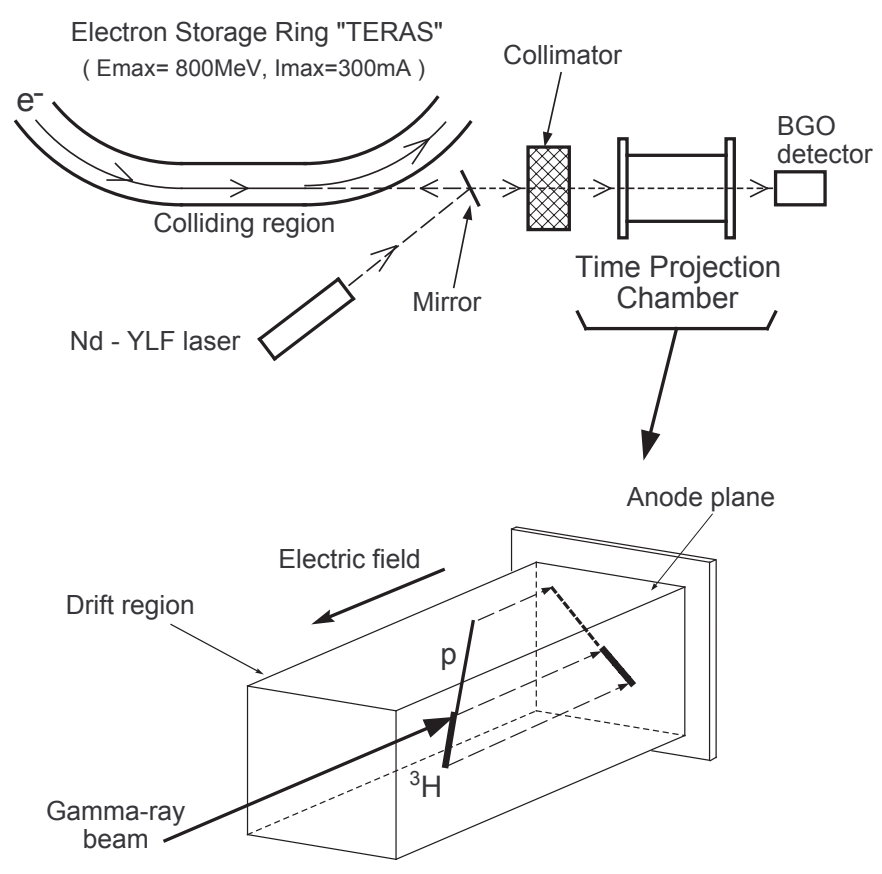

Figure 1: Experimental setup for measurement of photonuclear reaction using LCS $\gamma$-ray beam.

- considerably high intensity $\left(10^{3}-10^{5}\right.$ photons $\left./ \mathrm{MeV} / \mathrm{s}\right)$, and

$\cdot$ large polarization $(\sim 100 \%$, linear and/or circular $)$.

The above features of LCS $\gamma$-ray beams are great advantages for precise spectroscopic studies of the photonuclear reactions relevant for astrophysical nucleosynthesis.

\section{Time projection chamber with active target}

Since the energy regions of astrophysical interest are in general not so much higher than the threshold energies of the reactions, an active target is considered to be useful for detecting the reaction products with high efficiency. Therefore we employed a time projection chamber (TPC) whose operational gas serves as an active target. It enables us to measure low-energy charged particles from various reaction channels of the photodisintegration with detection efficiencies of nearly $100 \%$ and a solid angle of $4 \pi$. In addition, the TPC can measure the tracks and energy losses of the emitted charged particles. Those data are quite useful for precise identification of the true events and measurement of angular distributions of the reaction fragments. Detail of the present TPC is given in Ref. [8].

\section{Photodisintegration of ${ }^{4} \mathrm{He}$}

It has been claimed that the heating of the outgoing shockwave by delayed neutrinos would play the key role in Type-II SNes [3, 4, 5]. The interaction between neutrinos and nuclei is still one of the uncertain inputs for theoretical models of Type-II SNes. ${ }^{4} \mathrm{He}$ is considered as one of the 
principal ingredients of the matter surrounding the collapsing core [9], and therefore the neutrinoinduced nuclear reactions of ${ }^{4} \mathrm{He}$ rates would be of special importance. The neutrino-inelastic scattering reaction of ${ }^{4} \mathrm{He}$ is also supposed to make important contribution in production of light elements as well as heavy ones via the rapid process of nucleosynthesis in the neutrino-driven wind of Type-II SNe [6, 0, 10]. So it is necessary to obtain information about the responses of nuclei to neutrinos. Especially, nuclear responses to weak neutral current have been less understood mainly due to little experimental information. The photonuclear reactions will be a unique tool to investigate nuclear responses to weak neutral current because of the analogous structures between the operators of the weak interaction by neutral current and the electromagnetic interaction [2]. The cross section of the spallation reaction of ${ }^{4} \mathrm{He}$ by weak neutral current has been recently calculated in the energy region of supernova neutrinos $(\sim 8-12 \mathrm{MeV})$ by using a microscopic theory based on the Lorentz integral transform (LIT) method [11], which suggests 5-15\% enhancement in the reaction rate compared to the previous estimation with the systematic property of the giant dipole resonance [1]. Since those theoretical methods are also applied to photonuclear reactions, they can be tested by comparing the experimental data of the photonuclear reaction cross sections with the values estimated with those theories. So far the photonuclear reaction cross sections of ${ }^{4} \mathrm{He}$ have been measured using bremsstrahlung photons, tagged photons, pair-annihilation $\gamma$-rays, and LCS $\gamma$-rays, as well as the inverse ${ }^{3} \mathrm{H}(p, \gamma){ }^{4} \mathrm{He}$ and ${ }^{3} \mathrm{He}(n, \gamma){ }^{4} \mathrm{He}$ reactions. However, there have been discrepancy of $\sim 50-100 \%$ between the values measured with different experimental methods, and that requests further experimental efforts to measure the absolute photodisintegration cross sections of ${ }^{4} \mathrm{He}$ with more improved accuracy. Therefore we have performed a new measurement [12] using a LCS $\gamma$-ray beam at National Institute of Advanced Industrial Science and Technology (AIST) [13] and the above mentioned TPC.

In our experiment the systematic errors associating the experimental parameters in Eq. 2.1 are quoted as follows. The detection efficiency $\varepsilon$ for true photodisintegration events was evaluated to be better than $98 \%$ by comparing the measured pulse height spectra of the charged fragments with the Monte Carlo simulation. And also it was confirmed by measuring the photodisintegration of deuteron, whose cross section has been well known. Therefore the error of $\varepsilon$ is not greater than $2 \%$. The number density $n$ of the ${ }^{4} \mathrm{He}$ target was determined from the measured temperature and pressure of the TPC gas according to the equation of state of real gas, and its error is smaller than $0.2 \%$. The length $L$ of the effective region was defined from the position distribution of the reaction point of the true events along the $\gamma$-ray beam axis. Therefore its error amounts to $2 \%$, which is due to the accuracy of the position measurement. The incident $\gamma$-ray intensity was determined by the analysis of the $\gamma$-ray pulse height spectrum measured with a BGO scintillator whose intrinsic efficiency was larger than $99.5 \%$, and the error is less than $1 \%$. In summary, the total systematic error of the present method amounts to be less than $5 \%$.

Fig. 2 represents the measured cross section of the ${ }^{4} \mathrm{He}(\gamma, p){ }^{3} \mathrm{H}$ and ${ }^{4} \mathrm{He}(\gamma, n)^{3} \mathrm{He}$ reactions together with other available data, including the result of the most recent $(\gamma, n)$ experiment with bremsstrahlung photons [14], and the recent theoretical calculations. The present result is consistent with the previous data measured with monochromatic $\gamma$-ray beams. The present cross section of the ${ }^{4} \mathrm{He}(\gamma, n)^{3} \mathrm{He}$ reaction is in rather good agreement with the one obtained with the Faddeevtype calculation [16], while the present ${ }^{4} \mathrm{He}(\gamma, p)^{3} \mathrm{H}$ and ${ }^{4} \mathrm{He}(\gamma, n)^{3} \mathrm{He}$ cross sections are smaller 


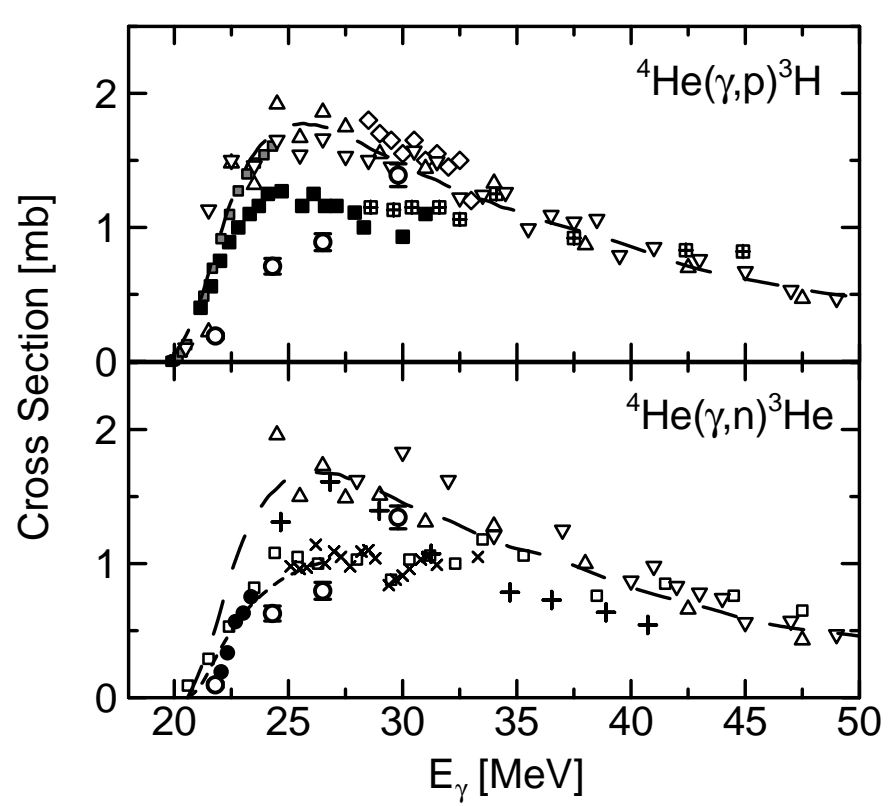

Figure 2: Two-body photodisintegration cross sections of ${ }^{4} \mathrm{He}$. The open circles denote the present data, while the other symbols indicate the previous ones (see the references in [12] $)$. Upper panel; $(\gamma, p)$ cross sections, open upward triangles; Gorbunov, open downward triangles; Arkatov et al., crossed squares; Bernabei et al., filled squares; Feldman et al., open diamonds; Hoorebeke et al., gray squares; Hahn et al. . Lower panel; $(\gamma, \mathrm{n})$ cross sections, open upward triangles; Gorbunov, open downward triangles; Arkatov et al., open squares; Berman et al., diagonal crosses; Ward et al., filled circles; Komar et al., crosses; Nilsson et al. . The error bars of the previous data are not shown for clarity. The long-dashed curves are the cross sections calculated using the LIT method with the MTI-III potential [17]. The short-dashed curve represents the calculated $(\gamma, \mathrm{n})$ cross section based on the AGS formalism [15, 16].

than the ones [17] calculated with the semirealistic Malfliet-Tjon (MT) nucleon-nucleon (NN) potential and the LIT method which was used in the calculation for the neutral current $v-{ }^{4} \mathrm{He}$ reaction rate in the energy region above $k T \sim 8 \mathrm{MeV}$ [11]. It should be noted a new calculation for the total photoabsorption on ${ }^{4} \mathrm{He}$ with the LIT method was performed using the realistic Argonne V18 NN potential and the Urbana IX three-nucleon force [18], and predicted a smaller peak cross section than the previous one with the semirealistic MT NN potential. Therefore further efforts in both theoretical and experimental studies of the photodisintegration of ${ }^{4} \mathrm{He}$ is quite important to investigate the role of the neutral current $v-{ }^{4} \mathrm{He}$ interaction in the neutrino heating in Type-II SNes.

\section{6. ${ }^{12} \mathbf{C}(\gamma, 2 \alpha)^{4} \mathbf{H e}$}

The ${ }^{12} \mathrm{C}(\gamma, 2 \alpha){ }^{4} \mathrm{He}$ reaction is interesting in connection with the $3 \alpha$ reaction, which plays a crucial role in stellar helium burning [19, 20, 21]. The existing experimental data are, however, quite scarce [22, 23, 24], and therefore we performed a new measurement in the similar manner as for the measurement of the photodisintegration of ${ }^{4} \mathrm{He}$ described above. Fig. 3 shows an example of the tracks of the ${ }^{12} \mathrm{C}(\gamma, 2 \alpha){ }^{4} \mathrm{He}$ reaction events detected with the present TPC. The true events can be accurately identified, because they are characterized by three tracks of the $\alpha$ particles emitted 


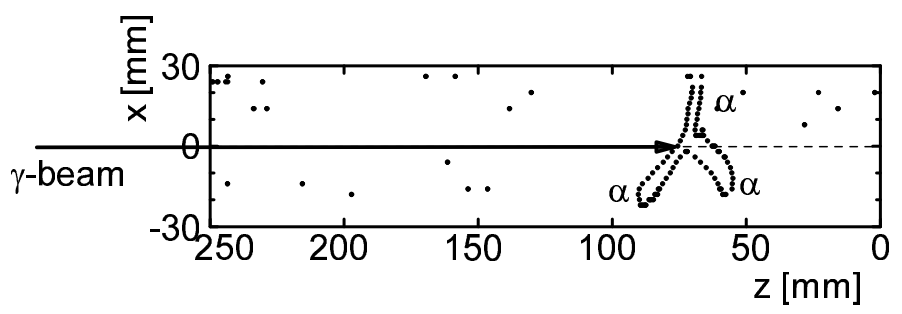

Figure 3: Example of the tracks of $\alpha$ particles emitted in the ${ }^{12} \mathrm{C}(\gamma, 2 \alpha){ }^{4} \mathrm{He}$ reaction events. The box shows the edge of the effective region of the TPC in section. The $\gamma$-ray beam axis is indicated by an arrow.

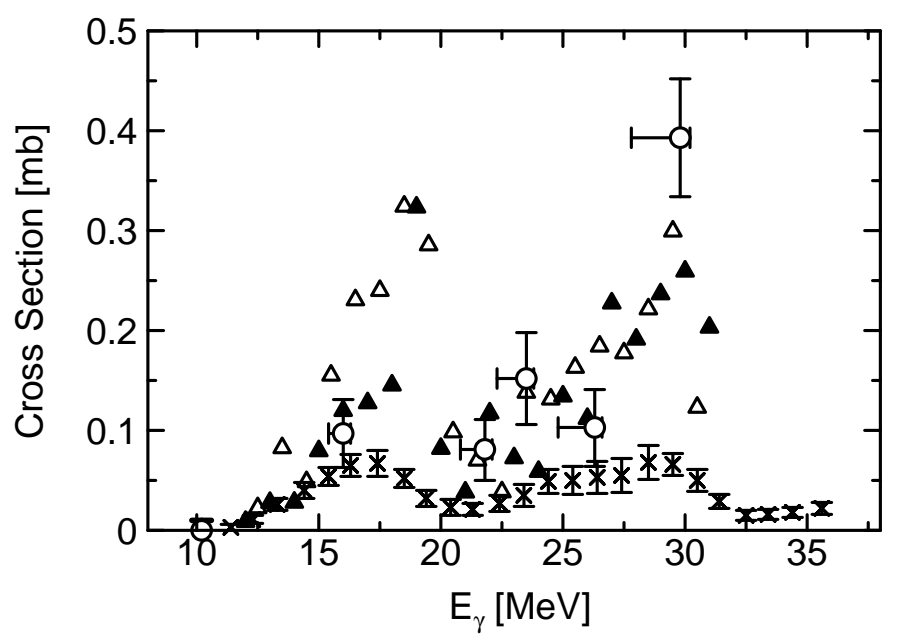

Figure 4: The ${ }^{12} \mathrm{C}(\gamma, 2 \alpha){ }^{4} \mathrm{He}$ reaction cross sections. Open circles; present work (preliminary), closed triangles; Maikov et al. [22], open triangles; Murakami et al. [23], diagonal crosses; Kotikov et al. [24].

at the reaction point on the $\gamma$-ray beam axis. Fig. 4 shows our preliminary result together with the previous ones. The previous three measurements were all performed with a similar method using bremsstrahlung photons and nuclear emulsion chambers, and the measured excitation functions are characterized by the peaks corresponding to the resonance excitations of ${ }^{8} \mathrm{Be}$ in the ${ }^{12} \mathrm{C}$ nucleus. The absolute value of the cross section from the most recent work is however smaller by a factor of $\sim 2-6$ than the values from two older measurements as shown in Fig. 4. Our result is found to be consistent with the older data by Maikov et al. [22] and Murakami et al. [23].

\section{Summary}

LCS $\gamma$-ray beams have a lot of advantages as mentioned above, and are considered to be useful for experimental studies of photonuclear reactions relevant for nuclear astrophysics. To perform high-precision measurements of photonuclear reactions, new LCS $\gamma$-ray sources with higher intensity and better quality have been developed at several new-generation synchrotron radiation facilities in the world. At the NewSUBARU of the University of Hyogo in Japan, a LCS $\gamma$-ray source in the energy of $16-38 \mathrm{MeV}$ has been newly developed by using the electron beam of 
$1.0-1.5 \mathrm{GeV}$ and the $1064 \mathrm{~nm}$ photons from a $\mathrm{Nd}: \mathrm{YVO}_{4}$ laser [25]. A unique feature of the $\gamma$-ray source at NewSUBARU is its excellent stabilities in the intensity and the energy distribution due to top-up mode operation, in which the electrons are continuously injected into the ring in order to keep the beam current constant. It is of great advantage in high-precision measurements of photonuclear reactions at low energies. And now it is planned to install a near-infrared laser based on the optical-parametric oscillation (OPO) technique [26, 27] in order to cover the $\gamma$-ray energy range from 5 to $85 \mathrm{MeV}$. The following experimental studies are currently ongoing or planned at the NewSUBARU LCS $\gamma$-ray facility;

(1) photodisintegrations of light nuclei relevant for few-nucleon physics and nuclear astrophysics,

(2) $(\gamma, \alpha)$ reactions relevant for $\alpha$-process and $\gamma$-process,

(3) $(\gamma, n)$ reactions of heavy nuclei relevant for p-process,

(4) isomers populated by $(\gamma, n)$ reactions in relation to heavy element synthesis and nuclear cosmochronology, and

(5) photo-excitations of isobaric analog states to simulate nuclear spin excitations by neutrinos.

\section{Acknowledgments}

The author would like to thank Profs. Y. Nagai, H. Ejiri, H. Utsunomiya, T. Mochizuki, and S. Miyamoto for fruitful discussions. This work was supported in part by Grant-in-Aid for Specially Promoted Research of the Japan Ministry of Education, Science, Sports and Culture and in part by Grant-in-Aid for Scientific Research of the Japan.

\section{References}

[1] S.E. Woosley, D.H. Hartmann, R.D. Hoffman, and W.C. Haxton, Astrophys. J. 356 (1990) 272.

[2] H. Ejiri, Phys. Rep. 338 (2000) 265.

[3] W.C. Haxton, Phys. Rev. Lett. 60 (1988) 1999.

[4] S.E. Woosley et al., Astrophys. J. 433 (1994) 229.

[5] H.-T. Janka and E. Muller, Astron. Astrophys. 306 (1996) 167.

[6] B.S. Meyer, Astrophys. J. 449 (1995) L55.

[7] A. Heger et al., Phys. Lett. B606 (2005) 258.

[8] T. Kii, T. Shima, T. Baba, and Y. Nagai, Nucl. Instr. Meth. in Phys. Res. A552 (2005) 329.

[9] K. Sumiyoshi et al., Astrophys. J. 629 (2005) 922.

[10] T. Yoshida, M. Terasawa, T. Kajino, and K. Sumiyoshi, Astrophys. J. 600 (2004) 204.

[11] D. Gazit and N. Barnea, Phys. Rev. C70 (2004) 048801.

[12] T. Shima et al., Phys. Rev. C72 (2005) 044004.

[13] H. Ohgaki et al., IEEE Trans. Nucl. Sci. 38 (1991) 386. 
[14] B. Nilsson et al., Phys. Lett. B626 (2005) 65.

[15] G. Ellerkmann, W. Sandhas, S. A. Sofianos, and H. Fiedeldey, Phys. Rev. C53 (1996) 2638.

[16] W. Sandhas et al., Nucl. Phys. A631 (1998) 210c.

[17] S. Quaglioni, W. Leidemann, G. Orlandini, N. Barnea, and V. D. Efros, Phys. Rev. C69 (2004) 044002 .

[18] D. Gazit, S. Bacca, N. Barnea, W. Leidemann, and G. Orlandini, Phys. Rev. Lett. 96 (2006) 112301.

[19] E.J. Öptic, Proc. Roy. Irish Acad. A54 (1951) 49; Mem. soc. roy. sci. Liége 14 (1953) 131.

[20] E.E. Salpeter, Astrophys. J. 115 (1952) 326; Annu. Rev. Nucl. Sci. (Annual Reviews, Inc., Stanford, 1953) Vol. 2 (1953) 41.

[21] S.M. Austin, Nucl. Phys. A758 (2005) 375c.

[22] V.N. Maikov, Zh. eksper. teor. Fiz. 34 (1958) 1406. translation: Sov. Phys. JETP 7 (1958) 973.

[23] A. Murakami, J. Phys. Soc. Japan 28 (1970) 1.

[24] E.A. Kotikov et al., Izv. Rossiiskoi Akademii Nauk, Ser. Fiz. 66 (2002) 445.

[25] K. Aoki et al., Nucl. Instr. Meth. in Phys. Res. A516 (2004) 228.

[26] J.J. Zayhowski, Opt. Lett. 22 (1997) 169.

[27] P. Gross et al., Opt. Lett. 27 (2002) 1433. 